This item was submitted to Loughborough's Research Repository by the author.

Items in Figshare are protected by copyright, with all rights reserved, unless otherwise indicated.

\title{
Nonlinear evolution of initially sine-like wedge acoustic waves
}

PLEASE CITE THE PUBLISHED VERSION

PUBLISHER

(C) IEEE

VERSION

VoR (Version of Record)

LICENCE

CC BY-NC-ND 4.0

REPOSITORY RECORD

Krylov, Victor V., A.P. Mayer, and D.F. Parker. 2010. "Nonlinear Evolution of Initially Sine-like Wedge Acoustic Waves". figshare. https://hdl.handle.net/2134/6160. 
This item was submitted to Loughborough's Institutional Repository (https://dspace.lboro.ac.uk/) by the author and is made available under the following Creative Commons Licence conditions.

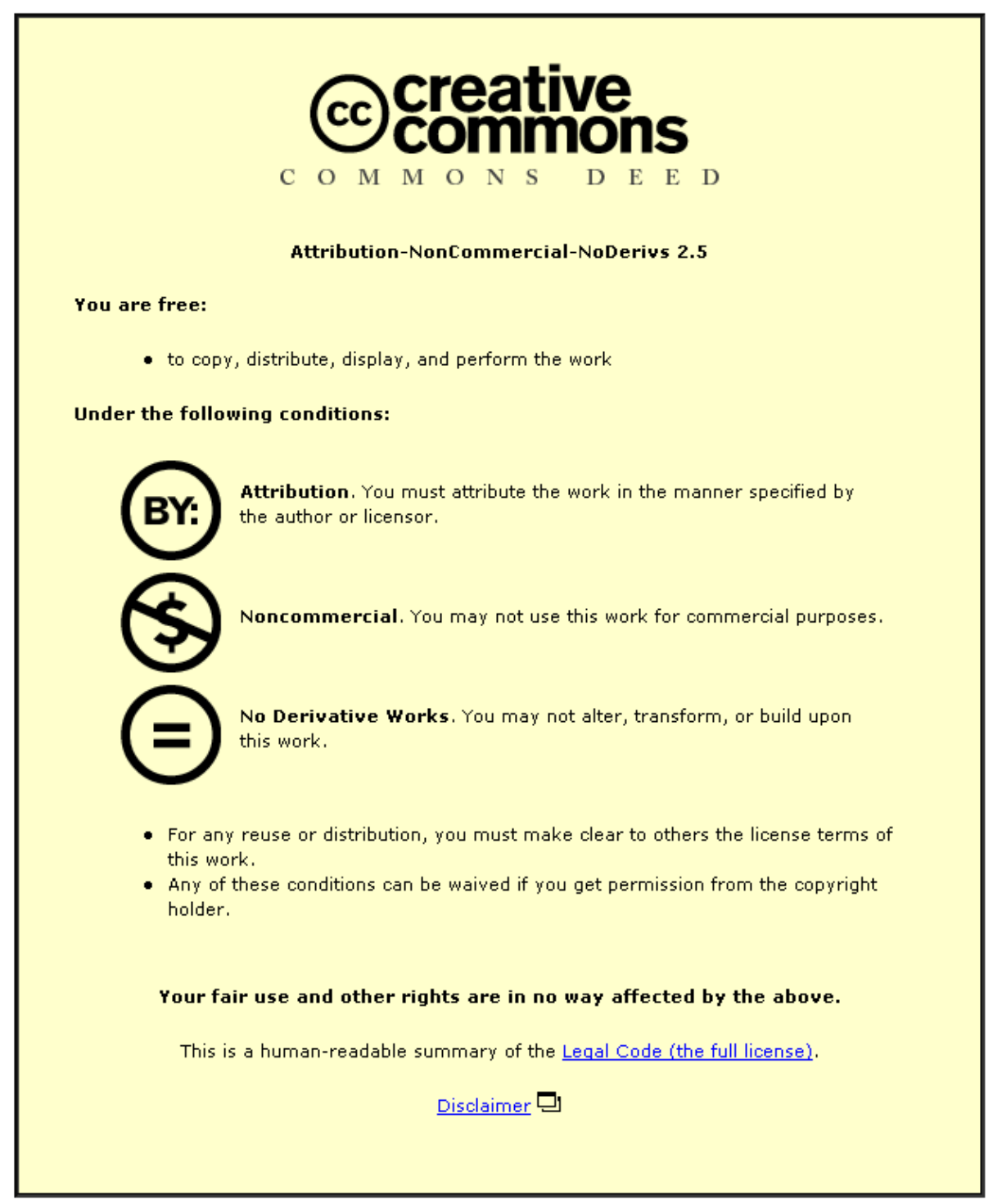

For the full text of this licence, please go to: http://creativecommons.org/licenses/by-nc-nd/2.5/ 


\title{
NONLINEAR EVOLUTION OF INITIALLY SINE-LIKE WEDGE ACOUSTIC WAVES
}

\author{
V.V. Krylov \\ Centre for Research into the Built Environment, The Nottingham Trent University, \\ Burton Street, Nottingham NGI 4BU, U.K.; \\ A.P. Mayer, \\ Institute of Theoretical Physics, University of Regensburg, \\ D-93040 Regensburg, Germany; \\ D.F. Parker, \\ Department of Mathematics and Statistics, University of Edinburgh, \\ Mayfield Road, Edinburgh EH9 3JZ, U.K
}

\begin{abstract}
In this paper the nonlinear behaviour of antisymmetric wedge acoustic waves propagating along the tip of a sharp elastic wedge is investigated theoretically. The nonlinear evolution equation is derived taking into account geometrical-acoustics approximation for wedge waves. In contrast to the case of surface acoustic waves for which the quadratic nonlinearity dominates, the lowest order of nonlinearity in this equation is cubic. For arbitrary propagation distances, the numerical solution taking into account 10 interacting wave harmonics has been carried out. The results show that an initially sine-like antisymmetric wedge wave distorts to a wave of trapezoidal form propagating with changed phase velocity.
\end{abstract}

\section{INTRODUCTION}

Along the tip of an elastic solid wedge, a special kind of mechanical vibration can propagate which is localised near the tip ${ }^{1-3}$. Such vibrations, usually called wedge acoustic waves, have a number of practical applications, especially in nondestructive testing of special engineering materials and in nonlinear signal processing devices 4,5 . The most interesting for applications are antisymmetric wedge waves, in particular waves in sharp-angle wedges (Fig. 1) for which the velocities of the antisymmetric modes can be very low.

Nonlinear behaviour of wedge acoustic modes is a matter of special interest because of the high concentration of elastic energy near the wedge tip and the absence of dispersion in ideal (nontruncated) wedges.

Earlier theoretical consideration of nonlinear effects in wedge acoustic waves was carried out by two of us 6 using a perturbation theory which is valid for relatively short propagation distances. However, it is important to investigate the nonlinear behaviour of wedge waves for arbitrary distances, as has been done in the last decade for Rayleigh surface waves ${ }^{7-9}$, Love waves 10 and BleusteinGulyaev waves 11 .

In the present work we consider the nonlinear behaviour of initially sine-like antisymmetric wedge acoustic waves using the nonlinear evolution equation approach. This takes into account all the interactions between a fixed number of harmonics and describes the evolution of wedge-wave profiles over large propagation distances.

\section{NONLINEAR EVOLUTION EQUATION}

In the geometrical-acoustics approximation for wedge acoustic waves considered here, the nonlinear wave equation has the following simplified form 6 :

$$
\begin{aligned}
& \frac{\partial^{2}}{\partial x^{2}}\left[a h^{3}(x)\left(\frac{\partial^{2} w}{\partial x^{2}}+\sigma \frac{\partial^{2} w}{\partial y^{2}}\right)\right]+2(1-\sigma) \frac{\partial^{2}}{\partial x \partial y}\left[a h^{3}(x) \frac{\partial^{2} w}{\partial x \partial y}\right]+ \\
& \frac{\partial^{2}}{\partial y^{2}}\left[a h^{3}(x)\left(\frac{\partial^{2} w}{\partial y^{2}}+\sigma \frac{\partial^{2} w}{\partial x^{2}}\right)\right]+\rho h(x) \frac{\partial^{2} w}{\partial t^{2}}+ \\
& 3 \frac{b}{a} x^{4} \Theta^{2}\left[2 \frac{\partial^{2} w}{\partial y^{2}}\left(\frac{\partial^{3} w}{\partial y^{3}}\right)^{2}+\left(\frac{\partial^{2} w}{\partial y^{2}}\right)^{2} \frac{\partial^{4} w}{\partial y^{4}}\right]=0
\end{aligned}
$$

Here $w$ is the displacement of the mid-plane of the wedge from equilibrium, $h(x)$ is the local thickness of the wedge, $a=E / 12\left(1-\sigma^{2}\right)=\rho c_{p}{ }^{2} / 12$, where $E, \sigma$ and $\rho$ are the Young's modulus, Poisson ratio and mass density of the 
wedge material, $c_{p}=2 c_{t}\left(1-c_{t}^{\left.2 / c_{1}^{2}\right)^{1 / 2}}\right.$ is the speed of a longitudinal wave in a thin plate (the lowest symmetric Lamb mode), $\quad b=0.4 f\left(c_{t} / c_{1}\right)^{6}$ is the quantity proportional to the nonlinear constant of the fourth order $f$ responsible for the term $f_{i j}\left(u_{i, j}\right)^{3}$ in the expansion of the elastic energy of an isotropic solid up to the fourth order in the strain tensor $10, y$ is the coordinate along the tip, $x$ is the coordinate directed inwards along the midplane of the wedge, and $\Theta$ is the wedge angle. For the sake of simplicity we take into account only one nonlinear modulus $f$ which is supposed to be much bigger than other nonlinear moduli of the fourth, third- and second orders. Thus, we do not consider here the effect of geometrical nonlinearity which may be predominant in real slender wedges 12 .

We recall that in the framework of the geometrical acoustic approximation the linear solution for the wedge modes has the form 6

$$
\begin{array}{r}
\mathrm{w}(\mathrm{x}, \mathrm{y}, \mathrm{t})=\frac{w_{0}}{\left(k _ { p ^ { x } / \Theta ) [ L ( x ) ] ^ { 1 / 2 } } \operatorname { c o s } \left[\mathrm{~L}(\mathrm{x}) \mathrm{k}_{\mathrm{p}} \mathrm{x} / \Theta\right.\right.} . \\
\left.\mathrm{n} \sin ^{-1} \mathrm{M}(\mathrm{x})-\pi / 4\right] \exp \left[\mathrm{i}\left(\sqrt{3} \mathrm{k}_{\mathrm{p}} \mathrm{y} / \mathrm{n} \Theta-\omega \mathrm{t}\right)\right],
\end{array}
$$

where $L(x)=\left[\sqrt{3}\left(2 \Theta / k_{p} x-\sqrt{3} / n^{2}\right]^{1 / 2}\right.$,

$$
M(x)=\left(1-\sqrt{3} k_{p} x / n^{2} \Theta\right),
$$

and $\mathbf{n}=1,23, \ldots$ is the mode number. The velocities of antisymmetric wedge modes $c=\omega / k$ are determined by the simple expression

$$
c=c_{p} n \Theta / \sqrt{3}
$$

Note that the geometrical-acoustics approximation for the case under consideration is valid for $k_{p} x / \Theta>1$. In this approximation, the wavefield does not penetrate into the region $x<x_{t}$ where $x_{t}=2 \sqrt{3} k_{p} / \Theta k^{2}$ determines the rayturning point or the location of the caustics.

Let us seek the solution of (1) in the form

$$
w(y, x, t)=\int_{-\infty}^{\infty} A\left(k, \varepsilon^{2} y\right) W(k x) e^{i k(y-c t)} d k,
$$

where $\mathbf{c}=\omega / \mathbf{k}$ is the velocity of a chosen wedge mode, $A(k)$ is the spectral density satisfying the condition $A(-k)$ $=A^{*}(\mathbf{k})$, and $W(k x)$ is the distribution of transverse displacements within a wedge acoustic mode and is determined from (2) rewritten in the form $w(x, y, t)=$ $W(k x) \exp [i(k y-(\omega) t)]$. As follows from (2), $W(k x)$ satisfies the condition $\mathrm{W}(-\mathbf{k x})=\mathrm{W}(\mathbf{k x})$.

Now, we substitute Eqn (4) into (1) and use $\int_{0}^{\infty} x W(k x) d x$ for both sides. Then, simplifying the linear part of the resulting equation, we obtain

$$
-\int_{0}^{\infty} \mathrm{xW}(\mathrm{kx}) \int_{-\infty}^{\infty} \mathrm{ik} \frac{d A\left(k, \varepsilon^{2} y\right)}{d y} 4 \mathrm{k}^{2} \mathrm{~W}(\mathrm{kx}) \mathrm{e}^{\mathrm{ik}(\mathrm{y}-\mathrm{ct}) \mathrm{dkdx}=}
$$$$
6 \frac{b}{a} \Theta^{2} \int_{0}^{\infty} x^{3} \int_{-\infty}^{\infty} k^{2} \mathrm{~A}\left(k, \varepsilon^{2} y\right) \mathrm{W}(\mathrm{kx}) \mathrm{e}^{\mathrm{ik}(\mathrm{y}-\mathrm{ct})} \mathrm{dk} *
$$

$$
\left\{\int_{-\infty}^{\infty}\left(-i k^{3}\right) * A\left(k, \varepsilon^{2} y\right) W(k x) e^{i k(y-c t)} d k\right\}^{2} d x-
$$

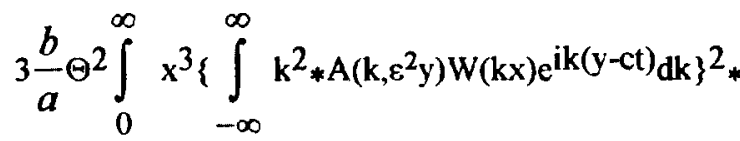$$
\int_{-\infty}^{\infty} k^{4} A\left(k, \varepsilon^{2} y\right) * W(k x) e^{i k(y-c t)} d k d x
$$

Using the convolution theorem, it is easy to derive from (5) the following nonlinear evolution equation:

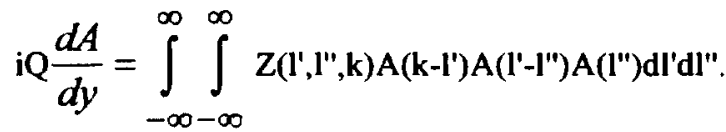

Here $Q=\int_{0}^{\infty} x W^{2}(k x) d x$ is the quantity proportional to the energy flow of a wedge wave, and the kernel $Z\left(l^{\prime}, 1^{\prime \prime}, k\right)$ is determined by the expression

$$
\begin{gathered}
\mathrm{Z}\left(\mathrm{l}^{\prime}, \mathrm{l}^{\prime \prime}, \mathrm{k}\right)=\frac{3 b}{4 a} \Theta^{2}\left(\mathrm{k}-\mathrm{l}^{\prime}\right)^{2}\left(\mathrm{l}^{\prime}-\mathrm{l}^{\prime \prime}\right)^{2}\left(\mathrm{l}^{\prime \prime}\right)^{3}\left(2 l^{\prime}-\mathrm{l}^{\prime \prime}\right) * \\
\mathrm{k}^{-3} \int_{0}^{\infty} \mathrm{x}^{3} \mathrm{~W}(\mathrm{kx}) \mathrm{W}\left(\left(\mathrm{k}-\mathrm{l}^{\prime}\right) \mathrm{x}\right) \mathrm{W}\left(\left(\mathrm{l}^{\prime}-\mathrm{l}^{\prime \prime}\right) \mathrm{x}\right) \mathrm{W}\left(\mathrm{l}^{\prime \prime} \mathrm{x}\right) \mathrm{d} \mathrm{x} .
\end{gathered}
$$


One should note that Eqn (6) has a form similar to that of the evolution equation for Bleustein-Gulyaev waves 11 where cubic nonlinearity is also paramount.

For small propagation distances $y$, the nonlinear evolution equation (6) has a power series solution which predicts the same growth of the third harmonic of a sinusoidal wedge wave as is predicted by the direct perturbational approach ${ }^{6}$. Indeed, if the spectral density $A\left(k, \varepsilon^{2} y\right)$ is described by

$$
A\left(k, \varepsilon^{2} y\right)=A^{\prime} \delta\left(k-k_{0}\right)+A \delta\left(k-3 k_{0}\right)
$$

where $\delta\left(k-k_{0}\right)$ is Dirac's delta-function, and $k_{0}=\omega / c$ is the wave-number of the first wedge harmonic, then substituting (8) into (6) and neglecting terms proportional to $A$ inside the integrals, one obtains, using the properties of the delta-function, the following equation:

$$
\mathrm{iQ} \frac{d A}{d y}=\mathrm{Z}\left(2 \mathrm{k}_{0}, \mathrm{k}_{0}, 3 \mathrm{k}_{0}\right) \mathrm{A}^{3} \text {. }
$$

After change of variable $\xi=(n / \sqrt{3}) k_{0} x=k_{p} x / \Theta$, this equation coincides with the corresponding perturbation equation 6 .

The solution of the evolution equation (6) for arbitrary values of $y$ can be obtained numerically. For this purpose, it is useful to transform the equation to facilitate carrying out the integration only over positive values of $\mathrm{l}^{\prime}$ and $\mathrm{l}^{\prime \prime} 8$.

Splitting the limits of the integration into $-\infty$ to 0 and 0 to $\infty$ and using the identity $\mathrm{A}(-\mathrm{k})=\mathrm{A}^{*}(\mathrm{k})$, one may rewrite equation (6) in the form

$$
\begin{aligned}
& \mathrm{iQ} \frac{d A}{d y}=\int_{0}^{\infty} \int_{0}^{\infty}\left[\mathrm{Z}\left(-\mathrm{l}^{\prime},-\mathrm{l}^{\prime \prime}, \mathrm{k}\right) \mathrm{A}\left(\mathrm{k}+\mathrm{l}^{\prime}\right) \mathrm{A}^{*}\left(\mathrm{l}^{\prime}-\mathrm{l}^{\prime \prime}\right) \mathrm{A}^{*}\left(\mathrm{l}^{\prime \prime}\right)+\right. \\
& Z\left(-l^{\prime}, l^{\prime \prime}, k\right) A\left(k+l^{\prime}\right) A^{*}\left(l^{\prime}+l^{\prime \prime}\right) A\left(l^{\prime \prime}\right)+ \\
& Z\left(l^{\prime},-l^{\prime \prime}, k\right) A\left(k-l^{\prime}\right) A\left(l^{\prime}+l^{\prime \prime}\right) A^{*}\left(l^{\prime \prime}\right) \quad+ \\
& \left.\mathrm{Z}\left(\mathrm{l}^{\prime}, \mathrm{l}^{\prime \prime}, \mathrm{k}\right) \mathrm{A}\left(\mathrm{k}-\mathrm{l}^{\prime}\right) \mathrm{A}\left(\mathrm{l}^{\prime}-\mathrm{l}^{\prime \prime}\right) \mathrm{A}\left(\mathrm{l}^{\prime \prime}\right)\right] \mathrm{dl} \text { 'dl" }
\end{aligned}
$$

Eqn (10) can then be rewritten in terms of positive values of the transform variables. For brevity we show the results only for the first integral in (10): $I_{1}$ (the other integrals $I_{2}, I_{3}, I_{4}$-are transformed in the same way):

$$
\mathrm{I}_{1}=\int_{0}^{\infty} d l^{\prime}\left[\int_{0}^{l} \mathrm{Z}\left(-\mathrm{l}^{\prime},-\mathrm{l}^{\prime \prime}, \mathrm{k}\right) \mathrm{A}\left(\mathrm{k}+\mathrm{l}^{\prime}\right) \mathrm{A}^{*}\left(\mathrm{l}^{\prime}-\mathrm{l}^{\prime \prime}\right) \mathrm{A}^{*}\left(\mathrm{l}^{\prime \prime}\right) \mathrm{d} \mathrm{l}^{\prime \prime}+\right.
$$

$$
\left.\int_{l^{\prime}}^{\infty} \mathrm{Z}\left(-\mathrm{l}^{\prime},-\mathrm{l}^{\prime \prime}, \mathrm{k}\right) \mathrm{A}\left(\mathrm{k}+\mathrm{l}^{\prime}\right) \mathrm{A}\left(\mathrm{l}^{\prime \prime}-\mathrm{l}^{\prime}\right) \mathrm{A}^{*}\left(\mathrm{l}^{\prime \prime}\right) \mathrm{dl} \mathrm{l}^{\prime \prime}\right] \text {. }
$$

Henceforth, we restrict attention only to periodic waveforms. The Fourier integral (4) may be replaced by the Fourier series:

$$
\begin{aligned}
w(x, y, t)=2 \sum_{n=1}^{\infty}\left\{C_{n} W\left(n k_{0} x\right) \cos \left[n k_{0}(y-c t)\right]-\right. \\
\\
\left.D_{n} W\left(n k_{0} x\right) \sin \left[n k_{0}(y-c t)\right]\right\},
\end{aligned}
$$

where $C_{n}$ and $D_{n}$ are real and imaginary parts of the Fourier coefficients $A_{n}$ respectively: $A_{n}=C_{n}+i D_{n}$. Taking this into account and using (11) along with the corresponding expressions for the integrals $\mathrm{I}_{2}, \mathrm{I}_{3}$ and $\mathrm{I}_{4}$, one can derive from (6) the infinite system of nonlinear differential equations versus $C_{n}$ and $D_{n}$ :

$$
\begin{aligned}
& \frac{d C_{p}}{d Y}=\mathbf{F}_{1}(\mathbf{C}, \mathbf{D}), \\
& \frac{d D_{p}}{d Y}=\mathbf{F}_{2}(\mathbf{C}, \mathbf{D}) .
\end{aligned}
$$

Here $F_{1}(C, D)$ and $F_{2}(C, D)$ are cubically-nonlinear matrix functions of vectors $\mathbf{C}$ and $\mathbf{D}$ with the components $C_{p}$ and $D_{p}$ respectively, $p=1,2,3, \ldots \infty$ (these functions are too bulky to be written here), $Y=k_{0} \mathrm{yg}^{2}$ is a nondimensional distance of propagation, $g=(3 / 4) b / a$, and a small parameter $\varepsilon$ is written in the form $\varepsilon=\mathrm{k}_{0} \mathrm{~A}_{\mathrm{ch}}$, where $A_{c h}$ is the characteristic amplitude of the wave.

\section{NUMERICAL CALCULATIONS AND DISCUSSION}

The numerical solution of the system of nonlinear equations (13) has been carried out by the modified Eulerian method with double precision. The system (13) was truncated, retaining non-zero $C_{p}, D_{p}$ only for $p \leq N$, with initial conditions at $\mathrm{Y}=0$ taken as the sine-like form: $D_{1}=1, D_{2} \ldots D_{N}=0$ and $C_{1} \ldots C_{N}=0$. Because the bulkiness of the equations (13) results in much computation time for every unit along $\mathrm{Y}$, the number of harmonics $\mathrm{N}$ has been chosen rather small $(\mathrm{N}=10)$. For the same reason, the number of terms in the process of numerical calculation of the integrals in kernels (7) inside $F_{1}$ and $F_{2}$ has been chosen to be $N_{i n t}=25$. The unit of iteration $\Delta \mathrm{Y}$ along the path of propagation $(\Delta \mathrm{Y}=0.005)$ was chosen as a compromise between the total calculation 
time and avoiding instabilities in numerical calculations at certain values of $\mathrm{Y}$, where all wave harmonics had explosion-like growth. The bigger the $\Delta Y$ step, the lower was the value of $Y$ at which the instability appeared. For $\Delta$ $Y=0.005$ the instability appeared at $Y=1.2$. As calculations show, this distance was enough to demonstrate visible distortion of the initial sine-like wave profile.

Fig. 2 shows the amplitudes of the third, fifth and seventh harmonics as functions of dimensionless distance $Y$. It is easily to see that for small $Y$ the third harmonic grows linearly with $Y$ (in agreement with the earlier results obtained by a perturbation approach 6 ), while the higher harmonics grow in a more complicated manner.

The distorted wave profiles near the tip plotted using the calculated values of $C_{n}$ and $D_{n}$ are displayed in Fig. 3 for several values of $Y$. One can see that, in contrast to the case of surface acoustic waves of Rayleigh type for which the quadratic nonlinearity dominates, the distorted wave profiles of wedge waves tend to the trapezoid-like form rather than to the saw-tooth form typical for surface waves. Another difference from the surface acoustic wave case is that distorted wedge waves possess a small change in velocity caused by self-interaction effects.

\section{REFERENCES}

1. Lagasse, P.E. Electronics Letters, 8 (1972) 372-373.

2. Maradudin, A.A., Wallis, R.F., Mills, D.L. \& Ballard, R.L. Phys. Rev. B6 (1972) 1106-1111.

3. Moss, S.L., Maradudin, A.A. \& Cunningham, S.L. Phys, Rev. B8 (1973) 2999-3008.

4. Krylov, V.V. Proc. II International Symp. on Surface Waves in Solids and Layered Structures, Vama, Bulgaria, 1989; World Scientific, Singapore (1990), p.174-189.

5. Adler, R., Hoskins, M., Datta, S. \& Hunsinger, B. IEEE Trans. on Sonics and Ultrasonics, 26 (1979) 345-347.

6. Krylov, V.V. \& Parker, D.F. Wave Motion, 15 (1992) $185-$ 200.

7. Kalyanasundaram, N. Int. J. Eng. Sci., 19 (1981) 279-286.

8. Parker, D.F. Int. J. Eng. Sci., 26 (1988) 59-75.

9. Maradudin, A.A. \& Mayer, A.P., in: Nonlinear Waves in Solid State Physics, eds.: Boardman, A.D., Twardowski, T. \& Bertolotti, M; Plenum, New York (1990), p. 113-161.

10 Kalyanasundaram, N. Int. J. Eng. Sci. 19 (1981) 287-293.

11. Mayer, A.P. Int. J. Eng. Sci. 29 (1991) 999-1004.

12. Mayer, A.P., Mozhaev, V.G., Krylov, V.V. \& Parker, D.F. Proc. NATO Advanced Research Workshop and Emil Warburg Symposium on Nonlinear Coherent Structures in Physics and Biology, Bayreuth, Germany, 1993.

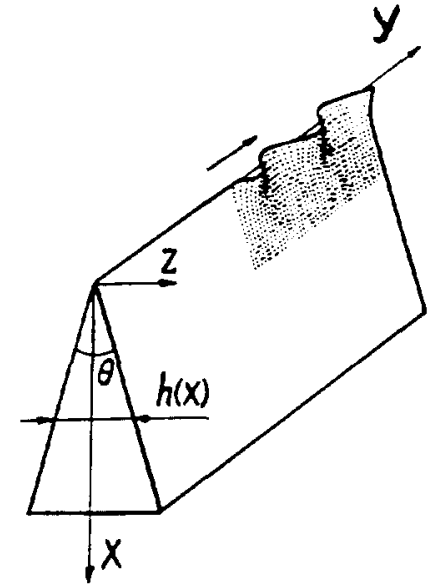

Fig.1. Geometry of the problem

$\left|A_{n}\right|$, Arbitrary Units

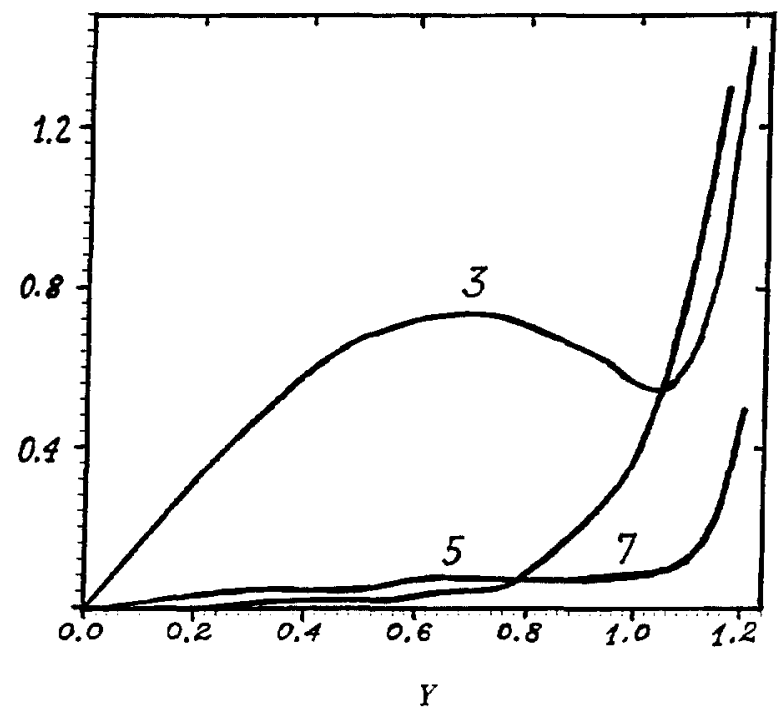

Fig.2. Amplitudes of harmonics as functions of $Y$

$\mathrm{W}(\Theta, 0,1)$, Arbitrary Units

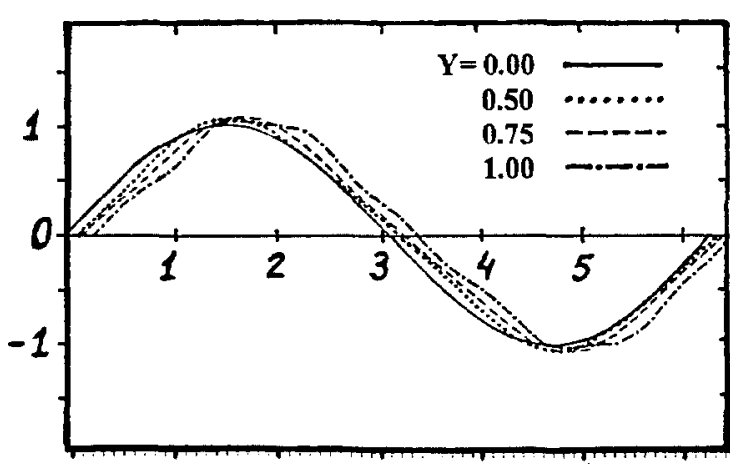

Fig.3. Distorted wave profiles; $\Theta=\mathbf{y}-\mathbf{c t}$ 